\title{
Aseguramiento y cobertura: dos temas críticos en las reformas del sector de la salud
}

\author{
Claudia Viviana Madies, ${ }^{1}$ Silvia Chiarvetti ${ }^{1}$ y Marina Chorny ${ }^{1}$
}

\begin{abstract}
RESUMEN La meta de salud para todos en el año 2000, que se estableció en Alma Ata hace más de 20 años, ha llevado a la adopción de reformas sectoriales en los países de América Latina y el Caribe encaminadas a ofrecer aseguramiento de salud a todos y cada uno de sus habitantes. Si bien las políticas adoptadas han llevado a adelantos importantes de carácter teórico y normativo, en la práctica las metas establecidas están lejos de haberse alcanzado y en muchos países existen grandes disparidades entre la cobertura real de la población y su cobertura teórica. Ello se debe, en gran medida, a la organización y otras características "endógenas" de los sistemas de salud vigentes en cada país, y a factores "exógenos" de carácter político, macroeconómico, social, epidemiológico y cultural.

En este documento se examinan detenidamente los diferentes tipos de sistemas de salud que existen actualmente en la Región y el efecto que ejercen sobre las fuentes de aseguramiento y cobertura de salud de los habitantes. Al final se presentan los distintos tipos de estrategias adoptadas por los países para extender la cobertura de salud, algunas veces mediante la focalización de las politicas en los grupos sociales más vulnerables.
\end{abstract}

Veintidós años han transcurrido desde que en la Reunión de Alma Ata, donde se fijó la meta de salud para todos en el año 2000, se establecieron los indicadores mínimos de salud que debían alcanzar los países de América Latina y el Caribe. Entre ellos figuraban el aumento de la esperanza de vida a 70 años; la reducción de la mortalidad infantil a 2,4 defunciones por 1000 nacidos vivos, y la extensión a cien por ciento de la población del acceso a agua potable y drenajes higiénicos para aguas

\footnotetext{
Fundación Isalud, Buenos Aires, Argentina. La correspondencia debe dirigirse a C.V. Madies a la siguiente dirección postal: Venezuela 925, Ciudad Autónoma de Buenos Aires, Argentina, C.P.1095. Correo electrónico: madiesc@isalud.com
}

residuales, así como a servicios de salud (1). Lamentablemente, el desarrollo político y social fue insuficiente; se acentuó la falta de equidad y hoy en día más de $20 \%$ de la población carece de acceso a la protección social de la salud (2).

Para comprender las dificultades que entrañan algunos de los procesos de reforma sectorial encaminados a alcanzar esas metas y a ampliar la cobertura del aseguramiento sanitario y el acceso a los servicios básicos de salud, no basta con examinar las políticas sociales que buscan extender dicha cobertura. También es preciso tener presente la estrecha vinculación entre estas políticas y los factores condicionantes y determinantes que dieron origen a las diferentes estrategias adoptadas por los países para alcanzar las metas.

\section{LOS PROCESOS DE REFORMA SECTORIAL Y LAS COBERTURAS TEÓRICA Y REAL}

En la Región de las Américas, la reforma del sector de la salud y el desarrollo de las políticas sanitarias se producen en un contexto de apertura económica, consolidación democrática y ajuste estructural del Estado (3), procesos que han influido en los mecanismos y estrategias orientadas a mejorar el acceso de los excluidos a sistemas de protección social.

En la década de los noventa se intensificaron estos procesos de reforma sectorial en numerosos países deseosos de proporcionar a todos sus habitantes servicios de salud eficientes y de buena calidad que respondieran a 
las necesidades acumuladas y emergentes de la población. En el sector de la salud se planteó la necesidad de alcanzar más equidad y eficiencia en la utilización de esos recursos, dirigiendo la atención a los grupos más vulnerables y a la resolución de problemas prioritarios.

Con estas políticas se han logrado adelantos importantes, fundamentalmente en el aspecto normativo o teórico, ya que los sistemas de salud de toda la Región reconocen, sin excepción y de manera implícita o explícita, el carácter universal del derecho a la salud (4), es decir, el derecho que a ella tiene la población en su totalidad (1). No obstante, las reformas adoptadas en este sentido no siempre han logrado en la práctica dar cobertura de salud a todos los habitantes.

Las estrategias para extender la provisión regular de servicios de salud a grupos sociales sin acceso a ellos o con acceso limitado (vale decir, sin cobertura real) se concentraron, en muchos casos, en los modelos de financiamiento y organización del sistema, relegando a un segundo plano los objetivos de mejorar la accesibilidad y calidad de las prestaciones (5). Los países que en el último quinquenio alcanzaron los mayores incrementos de la cobertura real fueron aquellos con sistemas de salud segmentados, que efectúan reformas sectoriales orientadas hacia una mayor integración de los seguros sociales de salud. En general estos países empiezan con altos porcentajes de exclusión y con un escaso desarrollo de las estructuras para usuarios dependientes del Estado, lo cual les confiere una mayor viabilidad política para el cambio (1). De ahí que el aseguramiento haya resultado una herramienta de gran utilidad para reducir la diferencia entre la cobertura teórica y la real.

La organización de los sistemas de salud ha tenido siempre, y sigue teniendo actualmente, una gran importancia a la hora de identificar las causas de la falta de acceso. La mayoría de los sistemas de salud de la Región, a excepción de los sistemas nacionales como los de Cuba y el Caribe de habla inglesa, presentan tres subsectores: el subsector público, que cubre fundamen-

\title{
La reforma del sector de la salud en Colombia: ¿un modelo de competencia regulada?
}

\author{
Francisco José Yepes Luján ${ }^{1}$ \\ y Luz Helena Sánchez Gómez²
}

En Colombia, entre las décadas de los ochenta y de los noventa tuvieron lugar dos procesos de reforma en salud que han provocado profundas transformaciones en el sector. En primer lugar, la descentralización política, administrativa y fiscal del aparato del Estado que se inició en los años ochenta y se concretó en el sector de la salud con la Ley 10/1990, y, en segundo lugar, la creación de un sistema general de seguridad social en salud con la Ley 100/1993. La confluencia de ambas reformas dificulta la separación de sus efectos, que en algunos casos son sinérgicos y en otros antagónicos.

La descentralización general corresponde a viejos anhelos de diversos actores sociales y se da en el marco de las negociaciones de paz con algunos grupos guerrilleros y en medio de las contradicciones de un sistema político que fluctúa entre el centralismo y el federalismo. La reforma de la seguridad social tiene lugar en el contexto de los procesos de apertura y globalización, de la necesidad de corregir defectos en eficiencia, equidad, cobertura, integralidad y calidad, y del desarrollo legislativo de la nueva Constitución de 1991.

\section{Antecedentes de la reforma de la seguridad social (RSS) en salud}

La RSS tiene dos antecedentes recientes: A) el Estudio Sectorial de Salud realizado en 1988-89 por el Ministerio de Salud y el Departamento Nacional de Planeación, que convocó a un muy amplio grupo de expertos para definir una imagen-objetivo

\footnotetext{
${ }^{1}$ Instituto de Seguros Sociales, Bogotá, Colombia. Toda la correspondencia debe dirigirse a Francisco José Yepes Luján, a la siguiente dirección postal: Instituto de Seguros Sociales, Av. 15 No. 100-43, Bogotá, Colombia. Correo electrónico: fcoyepes@openway.com.co

2 Asociación Colombiana de la Salud (ASSALUD), Bogotá, Colombia.
} 
del sector de la salud. Esa imagen planteó un sistema de cobertura universal, sin barreras económicas, geográficas ni culturales, que garantizara el derecho a la salud, con énfasis en la prevención de la enfermedad y la promoción de la salud, acciones coherentes con las necesidades municipales, la gestión comunitaria y las relaciones intersectoriales, y B) la Constitución de 1991, que consagró el derecho a la salud y a la seguridad social y dio pie a la formulación de la Ley 100/1993, que establece por primera vez en el país un sistema de seguridad social en salud con vocación explícita de cobertura universal, bajo los principios de equidad, solidaridad, eficiencia, calidad y participación social. Asimismo, la Constitución declaró al Municipio como la célula fundamental del ordenamiento territorial, haciendo más vinculante la dirección del cambio hacia la transferencia de competencias al poder local.

\section{La reforma}

La ley 100/1993 desarrolló los principios constitucionales y plasmó la RSS, que empezó a ser implementada a partir de enero de 1995.

El articulado final de la ley concilia las posiciones extremas favorables a la total privatización del sistema de salud y las favorables a un servicio nacional de salud estatal. El producto fue un sistema mixto donde el Estado asumió un papel rector y modulador, pero se liberó de las responsabilidades de aseguramiento y prestación de servicios que pasaron a ser asumidas, dentro de un esquema competitivo, por entidades públicas y privadas con y sin ánimo de lucro. La RSS creó dos regímenes: a) el contributivo, que se autofinancia con el aporte conjunto, obrero y patronal, de $12 \%$ del ingreso de los asalariados y de los trabajadores independientes con ingresos superiores a dos salarios mínimos (un punto porcentual de esa cantidad se transfiere al régimen subsidiado), y b) el subsidiado, financiado con los recursos de esta transferencia, más los recursos fiscales transferidos a los municipios vía la descentralización y los aportes que los municipios hacen de sus propios recursos.

La función directora y reguladora. La función de dirección y regulación está en manos del Ministerio de Salud, del Consejo Nacional de Seguridad Social en Salud (CNSSS) y de las administraciones territoriales (departamentos y municipios). "El Sistema General de Seguridad Social en Salud está bajo la orientación, regulación, supervisión, vigilancia y control del Gobierno nacional y del Ministerio de Salud..." (Ley 100/1993). Los niveles territoriales (departamentos, distritos y municipios) tienen talmente a los indigentes y que brinda prestaciones orientadas a la prevención, la curación y el saneamiento; el subsector de la seguridad social, que cubre a los trabajadores del sector formal y en algunos casos a sus dependientes; y el subsector privado, que generalmente atiende a los sectores de mayores recursos.

Por otro lado, mientras mayor es la articulación de los sistemas de salud, mayor es la disponibilidad de información con respecto a la cobertura real de la población, como en el caso de aquellos países que tienen sistemas nacionales de salud. Sin embargo, la escasez de información sobre la cobertura real no siempre implica que los países desconozcan la existencia de una falta de acceso a los servicios de salud. En la mayoría de estos países se emprendieron medidas para afrontar el problema, pero la falta de información ha impedido determinar su eficacia.

\section{Las causas de la disparidad entre la cobertura real y la teórica}

Las causas de la disparidad pueden clasificarse en exógenas y endógenas. Entre las causas exógenas, que son ampliamente conocidas, pueden identificarse las siguientes: 1) causas macroeconómicas (la pobreza, el desempleo, el subempleo, el trabajo informal, la distribución desigual del ingreso, los déficit fiscales); 2) causas políticas (los efectos perjudiciales de la reforma del Estado, la crisis del Estado benefactor, la inestabilidad política); 3) causas culturales (diferencias étnicas, analfabetismo); 4) causas sociales (diferencias de sexo, edad y otras características); 5) causas epidemiológicas; 6 ) causas geográficas. ${ }^{3}$

Para clasificar las causas endógenas, o propias del sistema, hay que analizar los modelos de atención de salud de cada país y su correlación con el tipo de cobertura que brindan. Siguiendo este criterio, se identificaron cuatro

\footnotetext{
Maceira D. Fragmentación e incentivos en los sistemas de atención en salud en América Latina y el Caribe. Banco Interamericano de Desarrollo; 1996. (Documento mimeografiado).
} 
grupos de países, cuya caracterización, perfil y causas de exclusión se describen a continuación.

\section{Países con sistemas públicos integrados}

Constituyen ejemplos de países con sistemas públicos integrados las Bahamas, Barbados, Costa Rica, Cuba, Granada, Jamaica y Trinidad y Tabago. Se trata de países con cobertura universal financiada sobre la base de impuestos y por asignación de presupuestos globales. En general, ofrecen la atención por medio de proveedores públicos de servicios. Costa Rica es la excepción, ya que cuenta con un subsector del seguro social coordinado con el subsector público, que actúa como modelo predominante de aseguramiento y provisión. El subsector privado siempre ha tenido un papel minoritario como complemento del aseguramiento público.

En estos países hay poca distancia entre la cobertura real y la teórica. Si se tiene en cuenta que cada uno de ellos se encuentra en una fase bastante avanzada de la transición epidemiológica, la población excluida se encuentra fundamentalmente entre los adultos mayores, algunos enfermos crónicos y los inmigrantes. No obstante, en Trinidad y Tabago un alto porcentaje de la población carece de acceso debido a factores geográficos, a la centralización del subsector público y a ineficiencias administrativas.

En general, las políticas y estrategias para extender la cobertura en este grupo de países están orientadas a mejorar la calidad de la prestación y se focalizan en los grupos vulnerables.

\section{Países con sistemas de aseguramiento mixto regulado}

Son ejemplos de sistemas de aseguramiento mixto regulado Argentina, Chile y Uruguay. Estos países se caracterizan por tener diversas formas de financiación, aseguramiento y provisión de servicios, con importantes grados de regulación pública. El subsector pú- funciones de dirección y organización de los servicios de salud en sus respectivos ámbitos y deben responder por el Plan de Atención Básica.

El CNSSS es un ente colegiado de concertación, creado por la Ley 100/1993 como organismo de dirección del sistema. En él participan el gobierno (Ministros de Salud, Hacienda y Trabajo), los entes departamentales y municipales de salud, los empleadores, los empleados, los profesionales de la salud, los usuarios del área rural, las entidades aseguradoras y las prestadoras de servicios. Dos decisiones de capital importancia que toma el CNSSS son la definición de la Unidad de Pago por Capitación y el contenido del Paquete Obligatorio de Salud, los dos grandes reguladores del sistema.

La competencia regulada en el aseguramiento y la prestación de servicios. La RSS liberó al Ministerio de Salud de la prestación directa de servicios de salud para dedicarse a la dirección, regulación, supervisión y control del sistema. Para ello la RSS generó unas entidades intermediarias encargadas del aseguramiento y de la conformación de sus redes de prestadores de servicios. Estas aseguradoras, denominadas Entidades Promotoras de Salud (EPS) para el régimen contributivo y Administradoras del Régimen Subsidiado (ARS) ${ }^{3}$ para el régimen subsidiado, ofrecen un seguro que consiste en un Paquete Obligatorio de Salud, determinado por el CNSSS, captan de los afiliados sus contribuciones al sistema ( $12 \%$ de sus ingresos), reciben del Fondo de Solidaridad y Garantía (FOSYGA) una Unidad de Pago por Capitación por cada afiliado y cada persona de su grupo familiar (cónyuge e hijos menores de 25 años) y contratan con una red de Instituciones Prestadoras de Salud (IPS) la entrega de servicios de salud a sus afiliados. Los recursos captados son del sistema, no de las EPS. Estas solo pueden apropiarse las unidades de capitación que les correspondan según el número de afiliados que documenten ante el FOSYGA. Para ello deben manejar los aportes de sus afiliados en cuentas especiales de las cuales solo pueden hacer retiros previa autorización del FOSYGA, una vez han documentado satisfactoriamente los pagos recibidos con los nombres e identificación de sus asegurados.

${ }^{3}$ Las ARS son una creación posterior a la ley 100/1993, con el fin de facilitar la expansión del régimen subsidiado. Tienen menos requisitos que las EPS y han proliferado con pequeño número de afiliados e ineficiencias notables. En la actualidad existe una política para aumentarles los requisitos y obligar a las pequeñas a consolidarse o desaparecer. 
Los hospitales de la antigua red pública de servicios de salud se transformaron en entes jurídica y administrativamente autónomos e iniciaron un proceso, aún en curso, de transformación de su financiamiento, desde el subsidio a la oferta al subsidio a la demanda. En otras palabras, se inició un proceso paulatino de disminución de los aportes directos del Estado y de su transformación en contratos con los entes aseguradores. De esta forma, la red pública entró a competir con el sector privado en la oferta de servicios. Actualmente las ARS deben contratar, como mínimo, $40 \%$ de su demanda con hospitales de la red pública, como una estrategia de protección de la misma.

Existe pues un sistema competitivo en el nivel de las aseguradoras, por una parte, y en el nivel de los prestadores, por otra. Las aseguradoras (EPS), delimitadas entre un ingreso per cápita predefinido por el CNSSS y un Paquete Obligatorio de Salud, deben competir en términos de eficiencia, a fin de reducir sus costos de operación, y en términos de calidad, a fin de atraer y retener afiliados. Las IPS, a su vez, deben competir entre ellas en iguales términos de eficiencia y calidad, a fin de captar usuarios y conseguir contratos con las EPS interesadas en minimizar sus costos de operación.

Inspección, vigilancia y control. La inspección, vigilancia y control del sistema corresponden a la Superintendencia de Salud. A ella le compete autorizar la constitución y funcionamiento de las EPS (29 en la actualidad), ARS (240), IPS (900) y Direcciones Departamentales y Locales de salud (1 134), y vigilar el cumplimiento de las normas, así como la adecuada aplicación de los recursos del sistema, entre otros. Las Direcciones Departamentales y Municipales de Salud también tienen responsabilidades de inspección, vigilancia y control en su ámbito territorial, pero sus competencias, recursos e instrumentos no están aún bien definidos, por lo cual, en la práctica, el sistema de inspección, vigilancia y control es todavía centralizado. Por otra parte, la capacidad operativa de la Superintendencia de Salud es muy limitada en términos presupuestarios y de recursos humanos, lo cual limita notablemente su capacidad de acción.

Los usuarios, organizados en asociaciones de usuarios tanto en el nivel de las aseguradoras (EPS) como de los prestadores (IPS), también deben contribuir a la vigilancia y control del sistema. Asimismo, existen los Comités de Veeduría Ciudadana, creados por la Constitución de 1991 para vigilar la inversión pública. blico cubre, en principio, a toda la población, aunque sus destinatarios son los grupos que no están cubiertos por los otros subsectores. El subsector del seguro social se financia con los aportes y contribuciones de los empleadores y trabajadores, y la provisión de servicios se realiza por conducto de instituciones privadas (en Chile, por ejemplo, por medio de las Instituciones de Salud Previsional: ISAPRE), o sindicales (como en Argentina, mediante las Obras Sociales) o estatales (por ejemplo, en Uruguay). La prestación de los servicios cubiertos por los seguros sociales alcanza a más de la mitad de la población en los tres países citados (1). El subsector privado está organizado y ofrece distintos planes de servicios de salud.

Las causas de la diferencia entre la cobertura teórica y la real se encuentran fundamentalmente en: 1) las deficiencias de los mecanismos de asignación y distribución de recursos, 2) la falta de coordinación y articulación entre los subsectores, 3) el gasto superior de los seguros privados y el seguro social en relación con el del subsector público y, 4) las dificultades que enfrentan las reformas sectoriales para superar la fragmentación del sistema y el sesgo de los seguros en favor de los cotizantes de mayores ingresos.

En estos países un número importante de personas que trabajan solo a tiempo parcial o en zonas rurales se encuentran desprotegidas por el seguro social. En Uruguay, a diferencia de Argentina y Chile, los familiares del titular del seguro no están protegidos por él. En Colombia se adoptó, como parte de la reforma sectorial, un modelo de competencia gerenciada que reconoce la financiación parapública y la provisión privada en el régimen contributivo, así como la financiación mixta (pública y parapública), y la provisión mixta (pública y privada) en el régimen subsidiado. Con ello se pretendía generar un incremento importante de la cobertura del aseguramiento en general, pero el proceso es aún muy reciente $\mathrm{y}$ no ha sido evaluado suficientemente como para incluir a este país en el grupo de países con aseguramiento mixto regulado. 


\section{Países con seguro de salud unificado}

En el Brasil la financiación es pública y la provisión es mixta. El sistema brasileño tiene un seguro social con tendencia a la unificación, debido a su modelo de financiamiento, su organización y la alta provisión privada de los servicios. Este país no optó por separar las entidades del seguro de salud, sino por unificarlas progresivamente hasta formar una entidad pública con metas de cobertura universal.

Las causas de la disparidad entre la cobertura teórica y la real radican en las fallas en la atención, las cuales generan un volumen importante del pago privado de bolsillo o mercados de seguros privados, que a veces se combinan con el aseguramiento público y que cubren a $20 \%$ de la población. Aunque se ha logrado incrementar la cobertura real, la falta de una mayor regulación impide lograr mejores resultados. Al mismo tiempo, la poca articulación entre los subsectores público y privado y la ausencia de una regulación adecuada ocasionan la sobrefacturación y el deterioro de la calidad de la prestación.

La población sin cobertura real está constituida por los sectores pobres y rurales, la población negra e indígena, los individuos sin empleo o con bajos ingresos, los niños menores de 3 años, los adultos mayores y las mujeres.

\section{Países con sistemas segmentados}

Son ejemplos de sistemas segmentados los de Bolivia, Ecuador, Guatemala, Guyana, Haití, Honduras, México, Nicaragua, Panamá, Paraguay, Perú, República Dominicana, Suriname y Venezuela. Este es un subgrupo heterogéneo de países en términos de financiación y provisión, pero se caracteriza por la profunda falta de integración entre sus distintos subsectores. Tal heterogeneidad también se ve reflejada en la cobertura real, muy limitada en Haití si se compara con la de Panamá o México.

Asimismo, las experiencias en curso para extender la cobertura varían ostensiblemente entre los países. Por ejemplo, en Panamá se crearon consor-
La descentralización y la seguridad social. La descentralización y la RSS son dos procesos impulsados por diferentes fuerzas y que obedecen a paradigmas distintos. Bien podrían transitar por vías separadas, por rutas contradictorias y enfrentadas o por caminos articulados y sinérgicos. En Colombia se han dado las tres situaciones. Así, entre 1993 y 1994, los subsidios a la demanda fueron inicialmente diseñados sobre la base del presupuesto nacional y del aporte de solidaridad del régimen contributivo como un camino alternativo a la descentralización y con un manejo administrativo centralizado desde el Ministerio de Salud. En 1995-96 su administración fue cedida a las Direcciones Seccionales de Salud en el nivel departamental. Finalmente, al ser recortados los aportes de la nación, dada la creciente presión derivada del déficit fiscal y la insuficiencia de los aportes de solidaridad del régimen contributivo, a partir de 1996 la financiación del régimen subsidiado se ató de manera creciente al proceso descentralizador. Una observación de la financiación municipal muestra que, en 1998 y 1999, por lo menos 50\% de la financiación provino de las contribuciones del FOSYGA y la otra mitad del esfuerzo municipal.

\section{¿Cuál es la situación cinco años después del inicio de la RSS?}

Entre los logros de la RSS cabe destacar:

El aumento de la cobertura del aseguramiento en salud hasta niveles nunca antes alcanzados. En el segundo semestre de 1997, la Encuesta Nacional de Calidad de Vida mostró niveles de aseguramiento de $57 \%$ de la población total del país (37\% en el régimen contributivo y $20 \%$ en el subsidiado), aun cuando probablemente durante los dos años siguientes ese nivel se haya deteriorado por la recesión económica y los altos niveles de desempleo que ha conllevado.

La cobertura de los más pobres alcanzó a $20 \%$ de la población a través del régimen subsidiado. Este segmento de población nunca antes había estado cubierto por ningún tipo de seguridad social y solo había podido acceder a los servicios de salud a través de la asistencia pública. Según el informe del CNSSS al Congreso de la República, la cobertura de la población con necesidades básicas insatisfechas era de $57 \%$ en $1998 .{ }^{4}$ La cobertura de $37 \%$ del régimen contributivo supone apenas un

\footnotetext{
4 Esta cifra proviene de la información suministrada al Minsiterio de Salud por los entes territoriales y puede ser una sobreestimación debido a la múltiple afiliación que ha sido detectada.
} 
aumento moderado sobre las coberturas de la seguridad social anteriores a la reforma (23\%), que se explicaría por la cobertura familiar del nuevo régimen, y hace sospechar que no se han incorporado nuevos cotizantes.

El Ministerio de Salud se liberó de la prestación directa de servicios y pudo asumir la función de formular políticas, dictar normas y dirigir y supervisar el sistema. Al mismo tiempo, la Superintendencia de Salud fue reformada para el cumplimiento de sus responsabilidades, pero a pesar de ello, está desbordada por sus nuevas competencias, debido a limitaciones presupuestarias $\mathrm{y}$ de recursos humanos.

El financiamiento de la salud aumentó en proporciones antes no vistas. Se estima que actualmente $10,5 \%$ del producto interno bruto (PIB) se destina a la salud. Como efecto del aumento de las contribuciones obreras y patronales ordenadas en la Ley 100/1993, el gasto parafiscal en salud pasó de 1,77\% del PIB en 1993 a 2,67\% en 1997. En 1990 los hospitales de primer nivel de la red pública incrementaron sus recursos en 2,6 veces, los de segundo nivel en 1,9 veces y los de tercer nivel en 1,5, lo cual significa un cambio en la tendencia histórica según la cual la mayor cantidad de recursos iba para los establecimientos de mayor complejidad.

Se está desarrollando en el país una conciencia de aseguramiento y de derecho a la salud que no había existido antes, en la medida en que las personas han empezado a incorporarse a los dos regímenes del seguro de salud y a exigir sus derechos, en muchas ocasiones incluso a través de los jueces.

Los principales problemas que persisten pueden resumirse en:

La evasión, entendida como la no afiliación al régimen contributivo de una parte importante de la población que debería estarlo, y la elusión, entendida como el pago de una contribución inferior a la corrrespondiente al nivel de ingresos, que se estima que están en el orden de $30 \%$. Ambas afectan de forma importante al equilibrio del sistema contributivo y al financiamiento del régimen subsidiado. A pesar de ello, aún no se han tomado medidas tendientes a buscar soluciones de fondo a este problema.

La falta de un sistema integrado de información que permita integrar las bases de datos de las distintas EPS y cruzar la información de afiliación y pago con impuestos nacionales $\mathrm{u}$ otras fuentes, que dificulta el control y facilita la evasión y el fraude. Un primer paso en este sentido acaba de ser dado por la Superintendencia de Salud al integrar por primera vez las bases de datos de afiliados a las EPS. cios entre el Ministerio de Salud y la Caja del Seguro Social; en México, se descentralizaron los servicios de la población no asegurada, que fueron delegados a los estados; y en Bolivia, Ecuador y Perú se impulsó desde el subsector público el desarrollo de esquemas de seguro para la población campesina o pobre.

En general, la distancia entre la cobertura real y la teórica se debe a: 1) la ineficiencia del subsector público en la asignación de fondos; 2) la fracción variable de la población cubierta por el seguro social; 3) la segmentación del seguro social en múltiples entidades de aseguramiento que comprometen su eficiencia $y, 4)$ el escaso desarrollo de los seguros privados, a pesar de su tendencia a la expansión. Dentro de este grupo de países, la exclusión es mayor donde la cobertura del seguro social es baja y la accesibilidad o calidad de los servicios públicos es deficiente. En estos casos, los sectores de bajos recursos deben optar por la cobertura de organizaciones no gubernamentales (ONG) o por la atención privada.

Por otra parte, la exclusión afecta fundamentalmente a la población rural de escasos recursos debido a la poca oferta de servicios, que se encuentran concentrados en las zonas urbanas y periurbanas. En suma, los países cuya oferta está mejor articulada y coordinada alcanzan los más altos niveles de cobertura, aun siendo bajo el gasto asignado al sector de la salud.

Esta articulación exige no solo la voluntad política, sino también el compromiso activo de todos los actores sociales, incluidos la sociedad civil, las ONG (6), los sindicatos y el sector privado, y el ejercicio del papel rector del Estado.

\section{ESTRATEGIAS PARA REDUCIR LAS DISPARIDADES}

Del análisis de los países de la Región, examinados en conjunto, se desprende que las políticas implantadas para extender la cobertura son fundamentalmente de dos tipos. Algunas reconocen directa y expresamente el carácter universal de la prestación; otras 
tienden a la focalización. Asimismo, dentro de dichas políticas se advierten distintas estrategias para extender la cobertura cuyo objetivo no siempre aparece claramente revelado. Las estrategias pueden clasificarse en los siguientes grupos (1):

- Estrategias destinadas a mejorar la eficiencia (atención primaria de la salud, participación de las ONG, creación del sistema nacional de salud, etc.). Por ejemplo, la atención primaria de la salud es una tendencia sostenida por todos los países. La participación de las ONG es importante en Argentina, Chile, Perú, Trinidad y Tabago y Uruguay.

- Estrategias para mejorar la productividad de los servicios: 1) fortalecimiento de la red de servicios (Ecuador, Honduras y Panamá); 2) paquete de servicios básicos (Argentina, El Salvador, Honduras, Jamaica, México, República Dominicana y Uruguay); 3) autogestión de hospitales (Argentina, Brasil y Chile); y estándares de calidad (Argentina, Barbados, Granada y San Vicente y las Granadinas).

- Estrategias orientadas a aumentar la participación local y a adaptar los servicios a las necesidades y condiciones locales (como los sistemas locales de salud en las Bahamas; la participación comunitaria en las Bahamas, Bolivia, Brasil, Chile, Costa Rica, Honduras, México, Nicaragua, República Dominicana, Suriname y Venezuela; y la descentralización en Argentina, Brasil, Ecuador, Granada, México, Panamá, República Dominicana, Trinidad y Tabago y Venezuela).

- Estrategias destinadas a reducir la exclusión financiera y cultural (aumento de la cobertura de la salud mediante el seguro social y la introducción de modelos de seguros básicos de salud; reforma de los modelos de pago y copago). Constituyen ejemplos Bolivia, Costa Rica, Ecuador, Granada, Jamaica, México, Nicaragua, Paraguay, República Dominicana, Trinidad y Tabago y Venezuela.

La debilidad de los mecanismos de control ciudadano, que ha facilitado el mal manejo de los instrumentos de focalización para la identificación de los pobres y ha permitido la inclusión indebida en el régimen subsidiado de personas que deberían pertenecer al régimen contributivo.

Las deficiencias de los sistemas de control por parte de la Superintendencia de Salud y de las administraciones territoriales, que facilitan la corrupción. ${ }^{5}$

La demora en el flujo de fondos de las aseguradoras hacia los proveedores, que ha generado una seria crisis en los hospitales, tanto públicos como privados. Estas demoras se han debido a diferentes causas, como el retardo de algunas administraciones municipales para cancelar a las ARS los recursos del régimen subsidiado o el retardo de las ARS y EPS para cancelar a los proveedores, en ocasiones para llevar a un máximo sus rendimientos financieros, y, en 1998 y 1999, la crisis financiera de la EPS del Instituto de Seguros Sociales. ${ }^{6}$

\section{Conclusiones}

En la RSS de Colombia encontramos elementos para hablar de un modelo de competencia regulada. La competencia entre aseguradoras se da en el marco delimitado por un ingreso per cápita definido (la Unidad de Pago por Capitación) y un pro-

\footnotetext{
5 Como la múltiple afiliación (ficticia) para cobrar varias veces la Unidad de Pago por Capitación, los cobros por personas no debidamente identificadas o el cobro de las IPS por servicios no prestados.

${ }^{6}$ Esta EPS es la mayor del sistema, con una cobertura de un poco más de $50 \%$ del régimen contributivo.
}

- Desarrollo de programas focalizados (programas de alimentación complementaria, extensión de los programas de vacunación, programas de ampliación de la cobertura a zonas rurales, programas de protección social para grupos de bajos ingresos). Todos los países sostienen este tipo de estrategias.

\section{CONCLUSIONES}

No cabe duda de que ofrecer cobertura de salud a todo ciudadano, sin excepción, constituye uno de los principios fundamentales de los procesos de reforma del sector de la salud en los países de la Región. Para lograrlo, los países aplican distintas modalidades 
ducto igual (el Paquete Obligatorio de Salud) $;{ }^{7}$ todas están en la obligación de aceptar a quien lo solicite y no se excluyen individuos previamente afiliados. Existe un listado de medicamentos esenciales que ordena un mínimo obligatorio. El rango de competencia queda limitado, por lo tanto, a la calidad del servicio ofrecido y a la eficiencia en la operación.

Persisten, sin embargo, problemas de articulación, y el papel de los diversos actores aún necesita una mayor definición, como en el caso de los entes territoriales en la función de inspección, vigilancia y control, mayor fortalecimiento operativo, como en el caso de la Superintendencia de Salud, o mayor desarrollo, como en el caso de la participación social. Con todo, la RSS colombiana es aún muy joven y necesita más tiempo para desarrollarse y madurar plenamente.

A pesar de la fuerte oposición de los grupos profesionales, en particular de los médicos, los beneficios de la RSS en términos de ampliación de la cobertura y mejoramiento del acceso a los servicios de salud, así como en la especialización de los diversos actores en funciones específicas (dirección, aseguramiento, prestación de servicios) hacen prever su permanencia. Hasta ahora no se han planteado dudas sobre su viabilidad financiera, aunque sí sobre su operatividad, que requiere ajustes que conduzcan a la solución de sus principales problemas. En este sentido, son tareas prioritarias el fortalecimiento de las funciones de inspección, vigilancia y control y de la participación social, el control de la corrupción, de la evasión y la elusión, y el desarrollo de un sistema integral de información.

\footnotetext{
7 La ley establece la transitoriedad de un Paquete Obligatorio de Salud más reducido para el régimen subsidiado. Este se inició en $50 \%$ del contributivo y se ha aumentado paulatinamente hasta $60 \%$. Sin embargo, la crisis económica del país hace prever que el igualamiento de los dos Paquetes Obligatorios de Salud se va a demorar más de lo previsto.
}

meta propuesta en Alma Ata. La concreción de los valores de universalidad, solidaridad, sostenibilidad y equidad, sobre los que se apoya la visión de una cobertura real del cien por ciento de la población, requiere su incorporación a las metas y acciones de la comunidad.

A esos efectos, debe considerarse que la evolución del Estado benefactor en los países de la Región (3), a excepción del Caribe no latino, se vio influenciada por el desarrollo temprano o tardío del seguro social (7). La creación de este seguro constituye un indicador de la capacidad de cada país para concretar el ejercicio del derecho a la salud enunciado legalmente. Por ende, los países con mayor exclusión son aquellos donde hubo un desarrollo tardío del seguro social, dificultades para alcanzar la integración del sistema, o ambos. En aquellos países donde las reformas del sector de la salud dieron prioridad a corregir esta situación, es donde se advierten los cambios más significativos.

La introducción o la extensión de seguros sociales de salud (8), seguros básicos adicionales o seguros para la cobertura de enfermedades catastróficas, así como la promoción del acceso universal a estos seguros, actuaron como mecanismos para reducir la exclusión social en el ámbito de la salud. En ese mismo sentido se han producido iniciativas locales, tales como los microseguros -el de Trenque Lauquen en Argentina es un ejemplo-, mediante las cuales se ha intentado ampliar la cobertura de los servicios de salud (9).

La gran variedad de estrategias desplegadas refleja la imposibilidad de identificar una única solución al crítico problema de la exclusión social en materia de salud. En las políticas y esfuerzos de los países para mejorar la efectividad del aseguramiento sanitario y garantizar el acceso equitativo y universal a los servicios de salud se deben tener en cuenta no solo los factores determinantes y condicionantes de carácter cultural, histórico, social, económico, político y local, sino también, los aspectos estructurales, de organización y de gestión del propio sistema de salud. 


\section{REFERENCIAS}

1. Office of Information Technology, Organización Panamericana de la Salud, Fundación ISALUD. Elementos para el análisis comparado de la extensión de la cobertura en salud en América Latina y el Caribe. Informe presentado en la Reunión Regional Tripartita, 29 de noviembre al 1 de diciembre de 1999, en México, D.F.

2. Office of Information Technology, Organización Panamericana de la Salud, Fundación ISALUD. Panorama de la exclusión de la protección social en salud en América Latina y el Caribe. Informe presentado en la Reunión Regional Tripartita, 29 de noviembre al 1 de diciembre de 1999, en México, D.F.

3. Filgueira F, Martínez J. Paradigmas globales y filtros domésticos: las reformas administrativas en políticas sociales en América Latina. Pittsburgh, Pensilvania: Universidad de Pittsburgh y Centro de Información y Estudios del Uruguay; 1999.

4. Organización Panamericana de la Salud. El derecho a la salud en las Américas. Washington, D.C.: OPS; 1989. (Publicación científica 509).

5. Teruel JR. Reforma del sector de la salud en las Américas. Informe presentado en el Seminario Internacional de Reforma del Sector de la Salud, 19 a 21 de febrero de 1996, en Lima, Perú.

6. Banco Interamericano de Desarrollo, Programa de las Naciones Unidas para el Desarrollo. Marco regulador de las organizaciones de la sociedad civil en Sudamérica. Washington, D.C. y Nueva York: BID y PNUD; 1997.
7. Mesa-Lago C. Atención de salud para los pobres de América Latina. Washington, D.C.: Organización Panamericana de la Salud, Fundación Interamericana; 1992. (Publicación científica 539).

8. Organización Panamericana de la Salud. La salud en las Américas. Washington, D.C.: OPS, 1998.

9. Organización Panamericana de la Salud, Organización Internacional del Trabajo. Síntesis de estudios de casos de microseguros y otras modalidades de protección social en salud en América Latina y el Caribe. Informe presentado en la Reunión Regional Tripartita, 29 de noviembre al 1 de diciembre de 1999, en México, D.F.
ABSTRACT

\section{Health insurance and health coverage: two critical issues within the context of health sector reform}

The goal of health for all in the year 2000, which was established at Alma Ata more than two decades ago, has led countries in Latin America and the Caribbean to adopt health sector reforms aimed at extending health coverage to each and every individual citizen. Whereas much has come about as a result of reform policies in the way of theory and legislation, in practice the goals that were established are far from attained, and many countries show large gaps in theoretical coverage on the one hand, and true coverage on the other. This is largely due to organizational features and other "endogenous" characteristics of the various countries' health systems, as well as to "exogenous" factors in the political, macroeconomic, social, epidemiologic, and cultural spheres.

This documents takes a close look at the different types of health systems that are currently operating in countries of the Region and their impact on sources of health insurance and health coverage for individuals living in those countries. The end of the article focuses on the different strategies adopted by the countries in an effort to extend health coverage, which in some cases involve policies targeting the most vulnerable social groups. 